\title{
The importance of including uric acid in the definition of metabolic syndrome when assessing the mortality risk
}

\author{
Nicola Riccardo Pugliese ${ }^{1}$ (1) - Alessandro Mengozzi ${ }^{1} \cdot$ Agostino Virdis $^{1} \cdot$ Edoardo Casiglia $^{15} \cdot$ Valerie Tikhonoff $^{14}$. \\ Arrigo F. G. Cicero ${ }^{2}$. Andrea Ungar ${ }^{4} \cdot$ Giulia Rivasi $^{4} \cdot$ Massimo Salvetti $^{3}$ - Carlo M. Barbagallo ${ }^{16} \cdot$ Michele Bombelli $^{10}$. \\ Raffaella Dell'Oro ${ }^{10}$. Berardino Bruno ${ }^{6} \cdot$ Luciano Lippa $^{7} \cdot$ Lanfranco D'Elia $^{11} \cdot$ Paolo Verdecchia ${ }^{17}$. \\ Francesca Mallamaci ${ }^{18}$. Massimo Cirillo ${ }^{13}$. Marcello Rattazzi ${ }^{19}$. Pietro Cirillo ${ }^{20}$. Loreto Gesualdo ${ }^{20}$. \\ Alberto Mazza ${ }^{21}$. Cristina Giannattasio ${ }^{22}$ - Alessandro Maloberti ${ }^{22} \cdot$ Massimo Volpe $^{23,24}$. Giuliano Tocci ${ }^{23,24}$. \\ Georgios Georgiopoulos ${ }^{25}$. Guido laccarino ${ }^{12}$. Pietro Nazzaro ${ }^{26}$. Gianfranco Parati ${ }^{8,9}$. Paolo Palatini ${ }^{15}$. \\ Ferruccio Galletti ${ }^{11}$. Claudio Ferri ${ }^{6}$. Giovambattista Desideri ${ }^{6}$. Francesca Viazzi ${ }^{5}$. Roberto Pontremoli ${ }^{5}$. \\ Maria Lorenza Muiesan ${ }^{3}$. Guido Grassi ${ }^{10}$. Stefano Masi ${ }^{1}$. Claudio Borghi ${ }^{2}$. The Working Group on Uric Acid and \\ Cardiovascular Risk of the Italian Society ofHypertension
}

Received: 2 October 2020 / Accepted: 1 February 2021 / Published online: 18 February 2021

(c) The Author(s) 2021

\begin{abstract}
Introduction Serum uric acid (SUA) has been depicted as a contributory causal factor in metabolic syndrome (MS), which in turn, portends unfavourable prognosis.

Aim We assessed the prognostic role of SUA in patients with and without MS.

Methods We used data from the multicentre Uric Acid Right for Heart Health study and considered cardiovascular mortality $(\mathrm{CVM})$ as death due to fatal myocardial infarction, stroke, sudden cardiac death, or heart failure.

Results A total of 9589 subjects (median age 58.5 years, $45 \%$ males) were included in the analysis, and 5100 (53\%) patients had a final diagnosis of MS. After a median follow-up of 142 months, we observed 558 events. Using a previously validated cardiovascular SUA cut-off to predict CVM $(>5.1 \mathrm{mg} / \mathrm{dL}$ in women and $5.6 \mathrm{mg} / \mathrm{dL}$ in men), elevated SUA levels were significantly associated to a worse outcome in patients with and without MS (all $p<0.0001)$ and provided a significant net reclassification improvement of $7.1 \%$ over the diagnosis of MS for CVM $(p=0.004)$. Cox regression analyses identified an independent association between SUA and CVM (Hazard Ratio: 1.79 [95\% CI, 1.15-2.79]; $p<0.0001$ ) after the adjustment for MS, its single components and renal function. Three specific combinations of the MS components were associated with higher CVM when increasing SUA levels were reported, and systemic hypertension was the only individual component ever-present (all $p<0.0001$ ).

Conclusion Increasing SUA levels are associated with a higher CVM risk irrespective of the presence of MS: a cardiovascular SUA threshold may improve risk stratification.
\end{abstract}

Nicola Riccardo Pugliese and Alessandro Mengozzi equally contributed.

Stefano Masi and Claudio Borghi joint last authorship.

Nicola Riccardo Pugliese

n.r.pugliese88@gmail.com

1 Department of Clinical and Experimental Medicine, University of Pisa, Via Roma, 67, 56126 Pisa, Italy

2 Department of Medical and Surgical Science, Hypertension and Cardiovascular Risk Factors Research Center, Alma Mater Studiorum University of Bologna, Bologna, Italy
3 Department of Clinical and Experimental Sciences, University of Brescia, Brescia, Italy

4 Department of Geriatric and Intensive Care Medicine, Careggi Hospital and University of Florence, Florence, Italy

5 Department of Internal Medicine, University of Genoa and Policlinico San Martino, Genoa, Italy

6 Department of Life, Health and Environmental Sciences, University of L'Aquila, L'Aquila, Italy

7 Italian Society of General Medicine, Avezzano, L'Aquila, Italy 


\section{Graphic abstract}
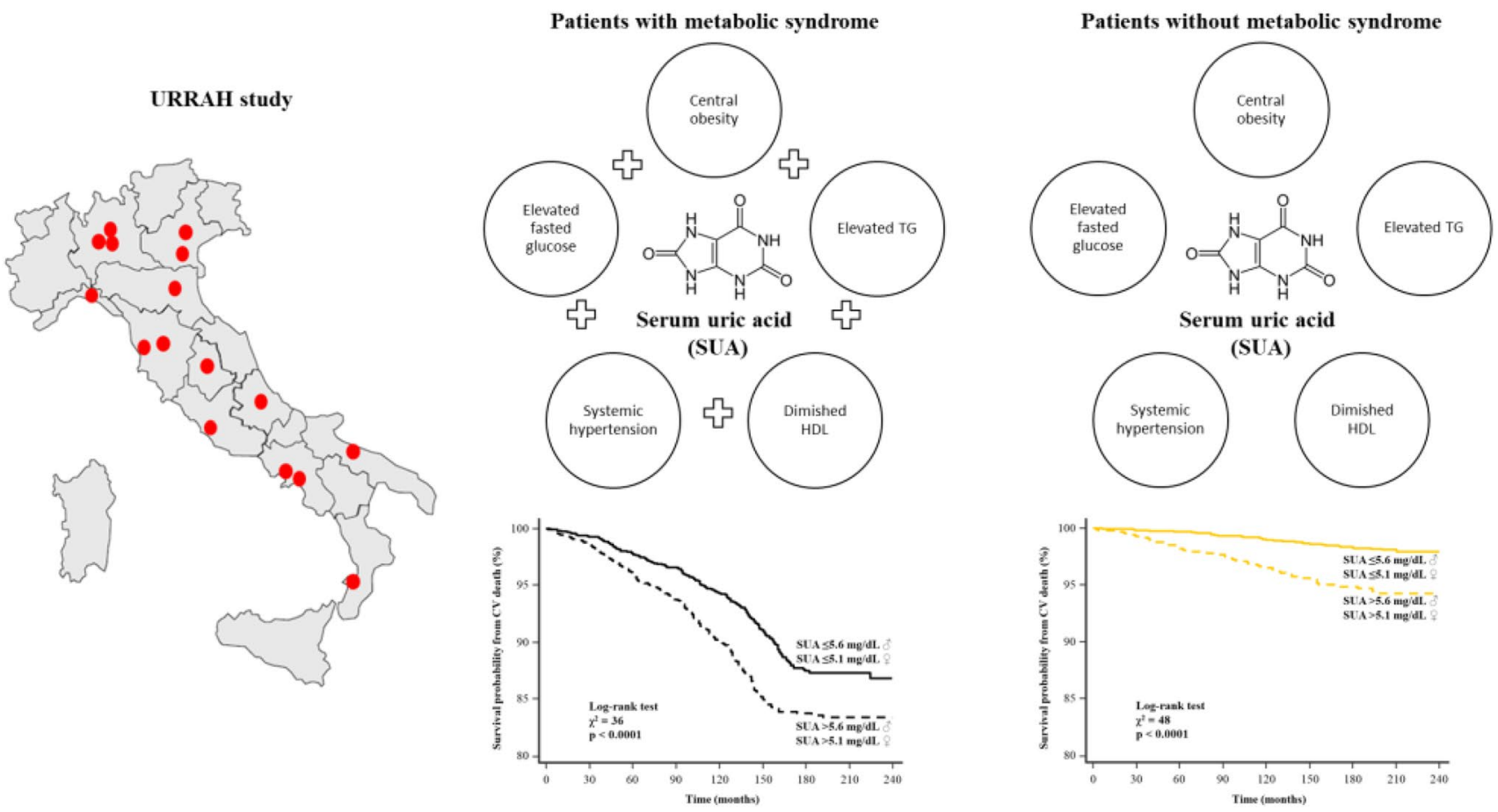

Keywords Serum uric acid $\cdot$ Metabolic syndrome $\cdot$ Cardiovascular mortality $\cdot$ Prognosis

\section{Introduction}

Cardiovascular disease (CVD) remains the leading cause of morbidity and mortality worldwide, and risk prediction remains the cornerstone of preventive medicine [1-3]. The metabolic syndrome (MS) represents a constellation of risk factors for CVD [4], and it is associated with a twofold increase in the risk of CVD, cardiovascular mortality (CVM), myocardial infarction and stroke, and a 1.5 -fold increase in the risk of all-cause mortality [5]. Increasing evidence suggests that uric acid may play a role in the development of MS, as hyperuricaemia belongs to a cluster of metabolic and haemodynamic abnormalities closely related to MS [4]. Several epidemiologic studies have reported a relation between serum uric acid (SUA) and CVD [6-9], as recognised by the latest European guidelines that recommend SUA evaluation in the stratification of the future cardiovascular risk of patients with arterial hypertension [3]. At the same time, little is known about the prognostic role of SUA in MS. The Working Group on SUA and cardiovascular risk of the Italian Society of Hypertension has specifically designed the URRAH project (Uric Acid Right for Heart Health) to study the relationship between SUA and CVD [10]. Using this extensive, prognostic registry, we investigated the role of SUA levels in improving further risk stratification of patients with MS.
Moreover, we assessed in which combination of MS parameters the addition of SUA provided the greatest improvement in the prediction of CVM risk. This is important as it has the potential to provide clear indications to the physicians on the type of metabolic patient in whom the assessment of SUA might lead to the greatest improvement in the stratification of the risk of CVM and should be, therefore, evaluated. Finally, we questioned if a cardiovascular SUA cut-off value lower than that currently used in clinical practice to define the risk of gout could be functional as a prognostic parameter in addition to MS diagnosis.

\section{Methods}

The Working Group on SUA and cardiovascular risk of the Italian Society of Hypertension has designed the URRAH project (Uric Acid Right for Heart Health) as a multicentre retrospective, observational cohort study, which involves data from several cohorts recruited within the Italian centres of hypertension and distributed in almost all the Italian regions. Datasets analysed during the current study are not publicly available but are available from the corresponding author on reasonable request. 


\section{Data collection}

As per protocol, SUA levels were collected from all the patients attending hypertension clinics at the time of the enrolment [10], together with information on cardiovascular risk factors where available. Anamnestic information, anthropometric measures (including waist circumference) and fasting blood lipid and glycaemic profiles were collected. Systolic and diastolic blood pressure was measured twice, in a quiet room, after five minutes resting and with the participant in sitting position. The second measure was used for all analyses. Hypertension was defined by the presence of at least two blood pressure recordings $>140 / 90 \mathrm{mmHg}$ or treatment with antihypertensive medications. Diabetes mellitus was defined by treatment with antidiabetic drugs, fasting plasma glucose $\geq 126 \mathrm{mg} / \mathrm{dL}$, or haemoglobin A1c $\geq 48 \mathrm{mmol} / \mathrm{mol}$. Renal function was evaluated through estimation of the glomerular filtration rate (eGFR), according to the Chronic Kidney Disease Epidemiology Collaboration equation [11]. We assessed MS according to the 2001 third report of the Adult Treatment Panel from the National Cholesterol Education Program [12]. A definite diagnosis MS was assigned in the presence of three or more of the following five cardiovascular risk factors: (1) central obesity (waist circumference: men $>102 \mathrm{~cm}$; women $>88 \mathrm{~cm})$; (2) elevated triglycerides $(\geq 150 \mathrm{mg} /$ $\mathrm{dL}$ ); (3) diminished high-density lipoprotein (HDL) cholesterol (men $<40 \mathrm{mg} / \mathrm{dL}$; women $<50 \mathrm{mg} / \mathrm{dL}$ ); (4) systemic hypertension ( $\geq 130 / \geq 85 \mathrm{~mm} \mathrm{Hg}$ ); and (5) elevated fasting glucose $(\geq 110 \mathrm{mg} / \mathrm{dL})$. From the overall population of the URRAH project $(n=22,714)$, we selected only the subjects in which all the MS criteria were available $(n=9589)$.

\section{Outcomes}

We considered cardiovascular mortality (CVM) at the end of the follow-up based on the following events: fatal events due to acute myocardial infarction, heart failure (HF) or stroke and sudden cardiac death. The definition of sudden cardiac death required documentation of significant arrhythmias or cardiac arrest. In case of death out of the hospital for which no autopsy was performed, sudden unexpected death was attributed to a cardiac cause. Information about death was obtained from hospital records or death certificates.

\section{Statistical analyses}

All tests were two-sided, with a $p$ value of $<0.05$ considered significant. Data were analysed with SPSS version 25.0 (IBM Corp., Armonk, NY) and R 3.6.2 (R Foundation for Statistical Computing, Vienna, Austria). Normally distributed continuous variables were presented as mean \pm SD and variables not following normal distribution as median (interquartile range). Differences in baseline characteristics were evaluated by independent samples $t$ test or Mann-Whitney $U$ test for continuous variables and $\chi^{2}$ test for nominal variables.

\section{Survival analyses}

Clinical follow-up data were censored at the time of the last visit or, for patients lost during follow-up, at the last date they were known to be alive. Kaplan-Meier survival curves for patients with and without MS were generated, and log-rank tests were used to assess differences between curves. Multivariable Cox proportional-hazards model was used to examine the association between MS and the outcome, including with stepwise forward selection SUA levels and all available clinical variables with biological plausibility selection (entry and removal value of $p<0.01$ and $p<0.10$, respectively). We tested interactions of SUA with age, gender (male), diabetes mellitus, eGFR and diuretics by incorporating corresponding interaction terms in the analysis. Associations are presented as hazard ratio (HR) with 95\% Cis and unstandardised $\beta$-regression coefficients (HR for CVM is relative to a one-unit change in the continuous variables included in the model). Variance inflation factor $>5$ was used to exclude multi-collinearity between selected variables. The association between different SUA levels and the outcome in patients with and without MS was analysed using restricted cubic splines with three knots and a reference SUA level of $4.1-5 \mathrm{mg} / \mathrm{dL}$. Analyses were adjusted for the covariates that were significant at the previous Cox regression to produce a smooth curve vs HR for CVM in the $y$-axis. We assessed the prognostic accuracy using the receiver operating characteristic (ROC) to calculate the area under the curve (AUC) and the cut-off point with the highest Youden index. As MS is defined by the presence of at least three out of five cardiovascular risk factors, 16 combinations are possible using binomial coefficients:

$\frac{5 !}{(5-3) ! \cdot 3 !}+\frac{5 !}{(5-4) ! \cdot 4 !}+\frac{5 !}{(5-4) ! \cdot 5 !}$

Therefore, we tested the interaction between each MS combination and SUA levels in predicting adverse events, to identify the MS combinations that are more associated with CVM when SUA is added to the model.

\section{Reclassification and discrimination analysis}

For estimating measures of reclassification and discrimination, we estimated the added value of identifying elevated SUA levels to predict the CVM using a model based on the presence of MS. Reclassification was deemed appropriate for participants with adverse outcome during clinical 
follow-up moving up in risk category and for participants without event moving down in risk category. Reclassification was summarised using continuous net reclassification index (cNRI) and integrated discrimination improvement (IDI) [13].

\section{Results}

The baseline characteristics of the studied population are reported in Table 1. A definite diagnosis of MS was observed in 5100/9589 (53\%) patients, which on average were older and more overweight, with a significantly higher frequency of the main $\mathrm{CV}$ risk factors and co-morbidities. As expected, blood tests showed a worse glycaemic and lipid profile in the subjects with MS. The most commonly prescribed medications were angiotensin receptor blockers, angiotensinconverting enzyme inhibitors, and dihydropyridine calcium channel blockers. Overall, drug therapy was more intense in those with MS. Supplemental Table 1 [Pugliese, Nicola Riccardo (2021): Supplemental material_rebuttal. figshare. Media. https://doi.org/10.6084/m9.figshare.13635062.v1] presents the same clinical characteristics as the population stratified by the previously validated cut-off of SUA levels for predicting CVM ( $0^{\lambda}>5.6 \mathrm{mg} / \mathrm{dL}$; $\left.+>5.1 \mathrm{mg} / \mathrm{dL}\right)[14]$.

During a median follow-up time of 142 months (interquartile range 60-163), a total of $558 \mathrm{CV}$ deaths were recorded (6\%), of which 175 were due to fatal myocardial infarction. Any adverse event was observed more frequently in patients with MS (Table 2), as confirmed by Kaplan-Meier analysis (Fig. 1a). The survival probability free from CVM for elevated and low SUA in the overall population was $87 \%$ and $94 \%$, respectively. The same analysis was conducted in patients with and without MS, showing high levels of SUA resulted in a worse outcome in both groups (log-rank test: all $p<0.0001$; Fig. $1 \mathrm{~b}, \mathrm{c}$ ). At the univariable Cox model, MS was associated with an increased risk of CVM (HR 5.21, 95\% CI 4.14-6.54; $p<0.0001$ ). This association remained highly significant even in a multivariable model adjusted for MS individual components, SUA levels, multiple CV risk factors and therapy: HR 2.25, 95\% CI 1.69-2.99); $p<0.001$ (Table 3). Five interaction terms were tested [SUA * age, SUA * gender (male), SUA * diabetes mellitus, SUA * eGFR and SUA * diuretics], and only SUA * age was significant when included in the model (HR $0.98,95 \%$ CI $0.97-0.99 ; p=0.03)$. Then, we confirmed the relation between MS, SUA and outcome after subdividing the population according to age [ $\geq$ and $<65$ years; Supplemental Tables 2-3, Pugliese, Nicola Riccardo (2021): Supplemental material_rebuttal. figshare. Media. https://doi. org/10.6084/m9.figshare.13635062.v1]. Likewise, when excluding 191 participants taking allopurinol, the results were not substantively different from the overall analysis [Supplemental Table 4, Pugliese, Nicola Riccardo (2021): Supplemental material_rebuttal. figshare. Media. https:// doi.org/10.6084/m9.figshare.13635062.v1]. Plotting the estimates from proportional-hazard modelling against SUA levels (reference level $4.1-5 \mathrm{mg} / \mathrm{dL}$ ) in patients with and without MS shows overlapping relative risks that increase for $\mathrm{SUA}>5 \mathrm{mg} / \mathrm{dL}$ and reach a plateau for values $>7$ (Fig. 2). Also, ROC analysis showed the prognostic accuracy of SUA was similar in patients with and without MS (AUC: 0.669 vs $0.694 ; p=0.1)$ but requiring different cut-offs (6.3 vs $5.6 \mathrm{mg} / \mathrm{dL}$ ).

Eight of the 16 combinations were representative of most of the cases of MS $(4272 / 5100,84 \%)$ and the most frequents were systemic hypertension + elevated triglycerides + diminished HDL $(885 / 5100,17 \%)$ and systemic hypertension + elevated triglycerides + elevated fasting glucose $(843 / 5100,16 \%)$. At Cox regression analysis for predicting CVM, the previous two combinations along with systemic hypertension + diminished HDL + elevated fasting glucose $(565 / 5100,11 \%)$ showed the highest $\beta$ regression coefficients after the interaction with SUA levels: $0.08 \pm 0.03,0.16 \pm 0.03$ and $0.18 \pm 0.03$ (all $p<0.0001$ ).

The addition of sex-specific elevated SUA levels $\left({ }^{1}>5.6 \mathrm{mg} / \mathrm{dL} ;\right.$; $\left.>5.1 \mathrm{mg} / \mathrm{dL}\right)$ to a model based on the presence of MS improved CVM classification: 47/588 patients with events (8\%) were reclassified correctly, while $11(2 \%)$ were reclassified incorrectly. At the same time, 632/9031 (7\%) patients without adverse outcome (i.e., CVM) underwent appropriate reclassification, while 305 (3\%) were reclassified inappropriately. In particular, reclassification analysis correctly reclassified $7.8 \%$ and $6.4 \%$ of subjects without and with MS, yielding a cNRI of $7.1 \%, p=0.004$ (Fig. 3). Discrimination was also improved, using the same cut-points, as indicated by IDI: $(4.6 \%, p=0.001)$. Reclassification analysis was further tested in different models, each based on the presence of the three MS combinations showing the most significant association with CVM after the interaction with SUA levels. Elevated SUA levels $(\hat{\jmath}>5.6 \mathrm{mg} / \mathrm{dL} ;$ $+>5.1 \mathrm{mg} /$ $\mathrm{dL}$ ) improved CVM reclassification (i.e., NRI) and discrimination (i.e., IDI) in all the models [Supplemental Table 5, Pugliese, Nicola Riccardo (2021): Supplemental material_rebuttal. figshare. Media. https://doi. org/10.6084/m9.figshare.13635062.v1]. The most significant improvement in predicting CVM was noted in MS combination including systemic hypertension + elevated triglycerides + diminished HDL $(\mathrm{cNRI}=8.3 \%, p=0.003$; IDI $=5.1 \% ; p=0.001)$. We tested the correlation analyses between SUA levels and the individual components of MS [Supplemental Table 6, Pugliese, Nicola Riccardo (2021): Supplemental material_rebuttal. figshare. Media. 
Table 1 Population characteristics in patients with and without metabolic syndrome (MS)

\begin{tabular}{|c|c|c|c|}
\hline Variable & MS $(n=5100)$ & Non-MS $(n=4489)$ & $p$ value \\
\hline \multicolumn{4}{|l|}{ Demographics } \\
\hline Age, years & $62 \pm 13$ & $52 \pm 16$ & $<0.0001$ \\
\hline Male & $1936(38)$ & $2351(52)$ & $<0.0001$ \\
\hline BMI, $\mathrm{kg} / \mathrm{m}^{2}$ & $29.1 \pm 4.3$ & $25.3 \pm 4.1$ & $<0.0001$ \\
\hline Waist circumference, $\mathrm{cm}$ & $100 \pm 11$ & $86 \pm 12$ & $<0.0001$ \\
\hline Family history of arterial hypertension & $2805(55)$ & $2245(50)$ & $<0.0001$ \\
\hline Family history of CVD & $1938(38)$ & $2200(49)$ & $<0.0001$ \\
\hline Current smoker & $1071(21)$ & $1077(24)$ & $<0.0001$ \\
\hline \multicolumn{4}{|l|}{ Clinical evaluation } \\
\hline Heart rate, beats/min & $72 \pm 12$ & $67 \pm 11$ & 0.2 \\
\hline Systolic blood pressure, $\mathrm{mmHg}$ & $153 \pm 21$ & $131 \pm 21$ & $<0.0001$ \\
\hline Diastolic blood pressure, $\mathrm{mmHg}$ & $88 \pm 12$ & $78 \pm 11$ & $<0.0001$ \\
\hline Arterial Hypertension & $4405(86)$ & $1985(44)$ & $<0.0001$ \\
\hline Diabetes mellitus & $1136(22)$ & $165(4)$ & $<0.0001$ \\
\hline CKD & 945 (19) & $323(7)$ & $<0.0001$ \\
\hline Gout & $90(2)$ & $5(0.1)$ & $<0.0001$ \\
\hline \multicolumn{4}{|l|}{ Blood tests } \\
\hline Haemoglobin, g/dL & $14.6 \pm 1.4$ & $14.2 \pm 1.5$ & $<0.0001$ \\
\hline Haematocrit, \% & $43 \pm 4$ & $43 \pm 4$ & 0.5 \\
\hline Total cholesterol, mg/dL & $217 \pm 40$ & $210 \pm 40$ & $<0.0001$ \\
\hline $\mathrm{HDL}, \mathrm{mg} / \mathrm{dL}$ & $43 \pm 12$ & $57 \pm 15$ & $<0.0001$ \\
\hline Triglycerides, mg/dL & $176(138-227)$ & $85(63-115)$ & $<0.0001$ \\
\hline Creatinine, $\mathrm{mg} / \mathrm{dL}$ & $0.98 \pm 0.2$ & $0.88 \pm 0.2$ & $<0.0001$ \\
\hline $\mathrm{eGFR}, \mathrm{mL} / \mathrm{min} / 1.73 \mathrm{~m}^{2}$ & $69 \pm 20$ & $84 \pm 21$ & $<0.0001$ \\
\hline Fasting blood sugar, mg/dL & $114 \pm 35$ & $88 \pm 15$ & $<0.0001$ \\
\hline Serum uric acid, mg/dL & $5.7 \pm 1.4$ & $4.8 \pm 1.2$ & $<0.0001$ \\
\hline Elevated serum uric acid ${ }^{\mathrm{a}}$ & $2599(51)$ & $820(18)$ & $<0.0001$ \\
\hline Azotemia, mg/dL & $35 \pm 11$ & $27 \pm 8$ & $<0.0001$ \\
\hline \multicolumn{4}{|l|}{ Therapy } \\
\hline ACE inhibitor & $3315(65)$ & $1571(35)$ & $<0.0001$ \\
\hline Angiotensin receptor blocker & $3519(69)$ & $1392(31)$ & $<0.0001$ \\
\hline DHP CCB & $663(13)$ & $225(5)$ & $<0.0001$ \\
\hline Non-DHP CCB & $51(1)$ & $45(1)$ & 0.8 \\
\hline Beta-Blocker & $612(12)$ & $359(8)$ & $<0.0001$ \\
\hline Allopurinol & $79(1.5)$ & $14(0.3)$ & $<0.0001$ \\
\hline Statins & $314(7)$ & $412(8)$ & 0.05 \\
\hline Diuretics & $605(12)$ & $285(6)$ & $<0.0001$ \\
\hline Hydrochlorothiazide & $226(12)$ & $135(5)$ & $<0.0001$ \\
\hline Indapamide & $37(2)$ & $33(1)$ & 0.02 \\
\hline Chlortalidone & $36(2)$ & $27(0.6)$ & $<0.0001$ \\
\hline Loop diuretics & $306(6)$ & $90(2)$ & $<0.0001$ \\
\hline
\end{tabular}

Values are mean \pm standard deviation, $n(\%)$, or median [25th quartile, 75 th quartile]

$A C E$ angiotensin-converting enzyme, $B M I$ body mass index, $C K D$ chronic kidney disease (eGFR $<60 \mathrm{~mL} /$ $\min / 1.73 \mathrm{~m}^{2}$ ), $C V D$ cardiovascular disease, $D H P C C B$ dihydropyridine calcium channel blocker, $e G F R$ estimated glomerular filtration rate, $H D L$ high-density lipoprotein, $S U A$ serum uric acid

a $\hat{0}>5.6 \mathrm{mg} / \mathrm{dL} ;$ ㅇ $>5.1 \mathrm{mg} / \mathrm{dL}$

$p$ values in bold indicate numbers that are significant on the $95 \%$ confidence limit 
Table 2 Clinical follow-up: cumulative event rates and Hazard Ratio for cardiovascular mortality in patients with and without metabolic syndrome

\begin{tabular}{lcclc}
\hline Event & MS $(n=5100)$ & Non-MS $(n=4489)$ & Hazard ratio (95\% CI)* & $p$ value* \\
\hline Cardiovascular death & $430(8.4)$ & $128(2.9)$ & $2.96(2.56-3.33)$ & $<\mathbf{0 . 0 0 0 1}$ \\
Fatal myocardial infarction & $140(2.7)$ & $35(0.8)$ & $2.87(1.75-5.59)$ & $<\mathbf{0 . 0 0 0 1}$ \\
Fatal stroke & $105(2.1)$ & $38(0.8)$ & $2.15(1.49-4.82)$ & $<\mathbf{0 . 0 0 0 1}$ \\
Sudden cardiac death & $58(1.1)$ & $25(0.6)$ & $1.57(1.19-3.76)$ & $\mathbf{0 . 0 1}$ \\
End-stage heart failure & $127(2.5)$ & $30(0.7)$ & $3.49(2.67-5.77)$ & $<\mathbf{0 . 0 0 0 1}$ \\
\hline
\end{tabular}

Legend as in the previous tables

*The hazard ratio is for the MS group as compared with the Non-MS group, and $p$ values were calculated by the log-rank test and are unadjusted for multiple variables

$p$ values in bold indicate numbers that are significant on the $95 \%$ confidence limit
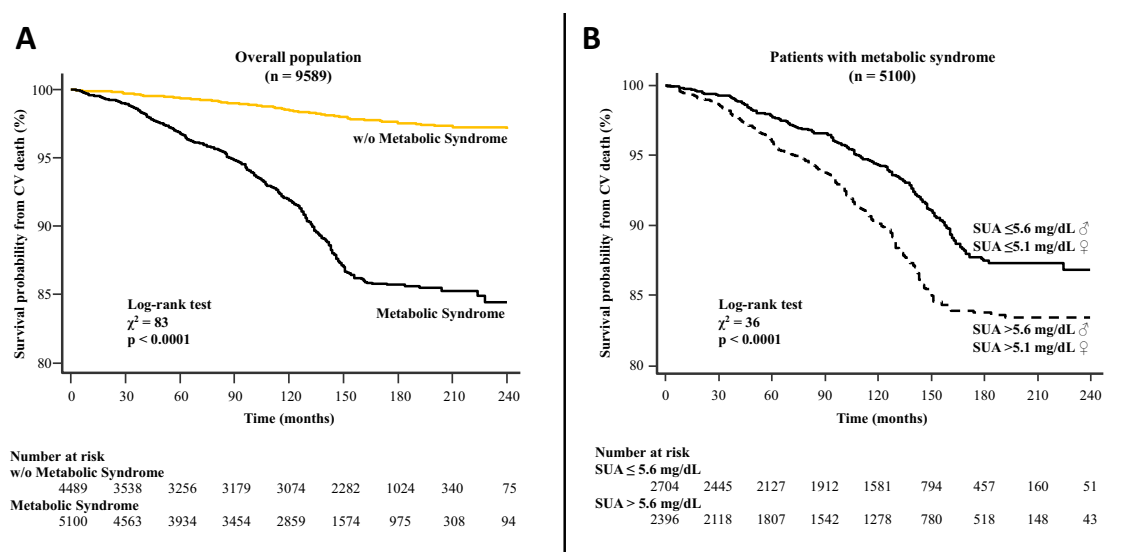

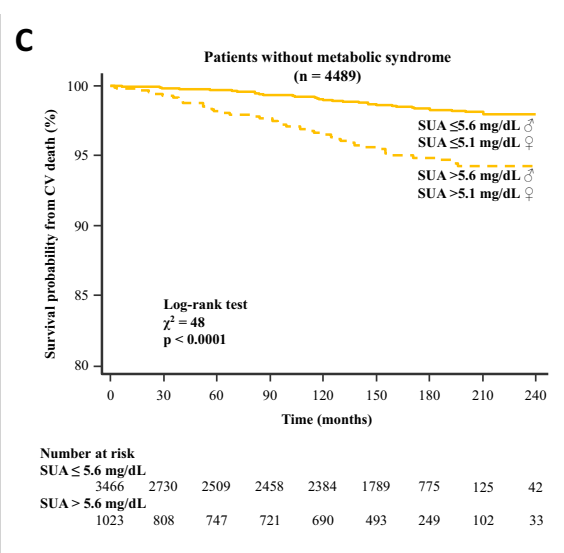

Fig. 1 Kaplan-Meier survival curves for cardiovascular death after a median follow-up of 18.5 months in the overall population (a), and patients with and without metabolic syndrome (b, c, respectively).
The patients are stratified according to serum uric acid into low $(\hat{\partial} \leq 5.6 \mathrm{mg} / \mathrm{dL} ;$ q $\leq 5.1 \mathrm{mg} / \mathrm{dL})$ and elevated levels $(\hat{\partial}>5.6 \mathrm{mg} / \mathrm{dL}$; q $>5.1 \mathrm{mg} / \mathrm{dL}$ ) https://doi.org/10.6084/m9.figshare.13635062.v1] and observed a significant correlation with all the variables (all $p<0.0001$ ): the highest correlation coefficient was observed with fasting glucose $(r=0.41)$ and triglycerides $(r=0.31)$.

\section{Discussion}

The present study suggests that the information on SUA levels refines the prediction of CVM obtained with MS and might help discrimination and reclassification of subjects at higher and lower CV risk [5].

The pathophysiological mechanism by which MS increases cardiovascular risk remains under debate [15]. Insulin resistance and central obesity are postulated to be the critical components of the metabolic syndrome, and both lead to glucose intolerance and dysglycemia [5]. Historically, elevated SUA levels in MS has been attributed to hyperinsulinemia, since insulin reduces renal excretion of uric acid [16]. Hyperuricemia, however, often precedes the development of hyperinsulinemia [17], obesity [18], and diabetes [19]. Independently on the cause-consequence relationship between SUA and MS, the presence of SUA in patients with MS seems to mark a greater risk of CVM. Indeed, animal models have shown that decreasing uric acid levels can prevent or reverse MS features [20], probably because hyperuricemia can induce endothelial dysfunction [21] and/or oxidative changes in adipocytes [22], which are typical stigmata of MS. Interestingly, all these features are increasingly described in HF with preserved ejection fraction (HFpEF), pointing to the existence of an inflammatory-metabolic phenotype [23]. A recent subanalysis from the PARAGON-HF trial demonstrated that hyperuricaemia was associated with an increased risk of adverse cardiovascular outcomes (CVM and HF hospitalisation) [24], suggesting that SUA may be a relevant therapeutic target also in HFpEF.

Our previous analysis documented that SUA levels are associated with all-cause mortality and CVM in hypertensive patients, independently of other CVD risk factors and the validated Heart Score risk algorithm. Also, the 
Table 3 Stepwise Cox proportional-hazards analysis for cardiovascular death

\begin{tabular}{|c|c|c|c|}
\hline Variable & Hazard ratio $(95 \% \mathrm{CI})$ & $p$ value & $\begin{array}{l}\beta \text { regres- } \\
\text { sion } \\
\text { coeffi- } \\
\text { cient }\end{array}$ \\
\hline Age, years & $1.14(1.09-1.18)$ & $<0.0001$ & 0.1 \\
\hline Male & $1.44(1.19-1.73)$ & 0.01 & 0.4 \\
\hline Arterial Hypertension & $1.59(1.17-2.15)$ & 0.003 & 0.5 \\
\hline Diabetes mellitus & $2.74(1.33-5.65)$ & 0.001 & 1.1 \\
\hline $\begin{array}{l}\text { Serum uric acid, mg/ } \\
\mathrm{dL}\end{array}$ & $1.79(1.15-2.79)$ & $<0.0001$ & 0.6 \\
\hline Metabolic syndrome & $2.25(1.69-2.99)$ & $<0.0001$ & 0.8 \\
\hline Statins & $0.33(0.21-0.52)$ & 0.001 & -1.1 \\
\hline SUA * age & $0.98(0.97-0.99)$ & 0.03 & 0.01 \\
\hline BMI, $\mathrm{kg} / \mathrm{m}^{2}$ & $0.97(0.95-1.02)$ & 0.1 & - \\
\hline Current smoker & $1.18(0.89-1.23)$ & 0.2 & - \\
\hline $\begin{array}{l}\mathrm{eGFR}, \mathrm{mL} / \mathrm{min} / 1.73 \\
\mathrm{~m}^{2}\end{array}$ & $1.17(0.89-1.29)$ & 0.2 & - \\
\hline Gout & $1.34(0.48-2.97)$ & 0.5 & - \\
\hline Haemoglobin, g/dL & $1.19(0.88-1.26)$ & 0.2 & - \\
\hline Haematocrit, $\%$ & $1.08(0.75-2.84)$ & 0.3 & - \\
\hline $\begin{array}{l}\text { Total cholesterol, } \\
\mathrm{mg} / \mathrm{dL}\end{array}$ & $1.01(0.99-1.02)$ & 0.3 & - \\
\hline $\mathrm{HDL}, \mathrm{mg} / \mathrm{dL}$ & $0.99(0.98-1.01)$ & 0.1 & - \\
\hline Triglycerides, mg/dL & $1.01(0.99-1.01)$ & 0.5 & - \\
\hline Diuretics & $1.43(0.82-3.63)$ & 0.1 & - \\
\hline SUA * gender (male) & $1.04(0.93-1.17)$ & 0.1 & - \\
\hline $\begin{array}{l}\text { SUA * diabetes mel- } \\
\text { litus }\end{array}$ & $0.95(0.85-1.08)$ & 0.4 & - \\
\hline SUA $*$ eGFR & $0.87(0.79-1.14)$ & 0.5 & - \\
\hline SUA * diuretics & $1.05(0.91-1.22)$ & 0.5 & - \\
\hline
\end{tabular}

Legend as in the previous tables

$p$ values in bold indicate numbers that are significant on the $95 \%$ confidence limit association of SUA with all-cause mortality and CVM was continuous [14]. The present analysis focuses on the role of uric acid in patients with MS, which represent a population at high risk of adverse CV events [5]. Krishnan et al. have analysed the incidence of myocardial infarction in 12,866 men at high risk of adverse coronary events and found a significant risk relationship with hyperuricemia ( $\geq 7 \mathrm{mg} / \mathrm{dL}$ ) that was independent of renal function, diuretic use and MS [25]. The present study provides further insights on the topic, because we proved that higher SUA level portends a worse outcome regardless of the presence of MS or other common CV factors, including interaction with diuretic intake. Noteworthy, we enrolled a population with an intermediate risk of adverse $\mathrm{CV}$ events according to Heart Score risk charts. Nevertheless, when we analysed SUA levels as a continuous variable in MS and nonMS, we observed overlapping risk curves that raise when SUA $>5 \mathrm{mg} / \mathrm{dL}$ and reach a plateau for values $>7 \mathrm{mg} / \mathrm{dl}$. Indeed, the prognostic accuracy of SUA levels was similar in the two groups, supporting the use of a cardiovascular threshold of SUA [14] to improve discrimination and reclassification of CVM in patients with and without MS.

\section{Clinical perspectives}

The present study confirms the importance of implementing SUA dosage in clinical practice for a more precise CV risk stratification also in patients with MS, of which hyperuricemia was formerly a part [26]. In particular, we identified some specific combinations of the MS individual components associated with significantly higher CVM when increasing SUA levels are reported, i.e., systemic hypertension with two between elevated triglycerides, diminished HDL and elevated fasting glucose. Interestingly, the most significant improvement in predicting CVM was noted in the most common MS combination (i.e., systemic hypertension + elevated triglycerides + diminished HDL),
Fig. 2 Hazard ratio for cardiovascular $(\mathrm{CV})$ death across the range of the serum uric acid levels (reference value $4.1-5 \mathrm{mg}$ / $\mathrm{dL}$ ) in patients with and without metabolic syndrome. Estimates are from proportional hazard modelling as a restricted cubic spline function of SUA levels and dotted lines show $95 \%$ confidence intervals. Analyses were adjusted for age, gender, arterial hypertension, diabetes mellitus and statin intake

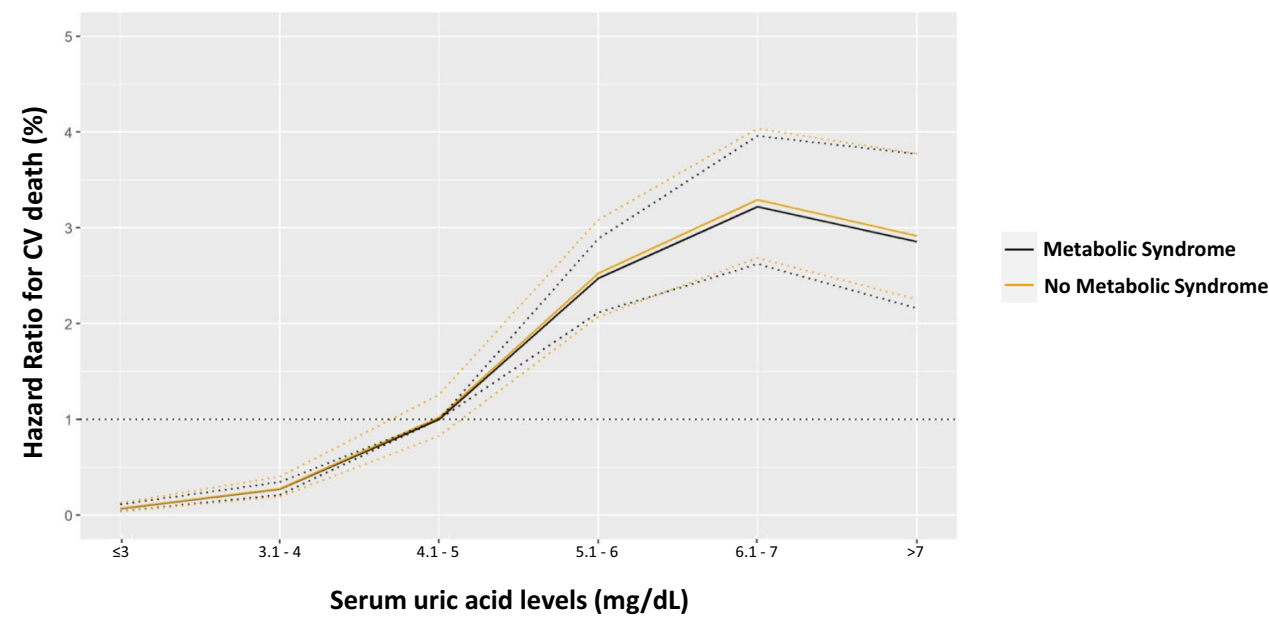




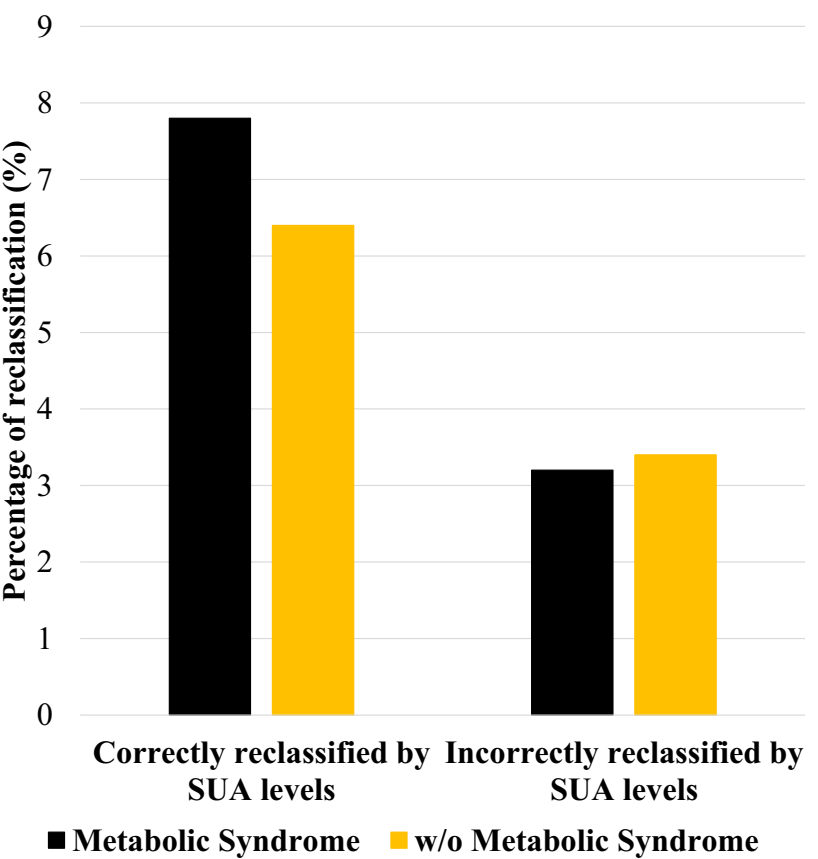

Fig. 3 Reclassification analysis. Percentage of patients with and without metabolic syndrome correctly and incorrectly reclassified for event risk prediction when elevated serum uric acid (SUA) levels $\left({ }^{\lambda}>5.6 \mathrm{mg} / \mathrm{dL} ;\right.$ ㅇ $\left.>5.1 \mathrm{mg} / \mathrm{dL}\right)$ were added to the model

encouraging the adoption of a cardiovascular threshold of SUA in clinical practice. As several Mendelian randomisation studies have suggested that the association between SUA and CVM is likely to be influenced by pleiotrophy [27-29], understanding the complex relationship between SUA and other CVD risk factor is critical. Moreover, it might help the identification of patients who could experience the most significant benefits from urate-lowering treatments and, as such, refine the inclusion and exclusion criteria in randomised clinical trials [26]. Indeed, a careful assessment of the clinical characteristics of the patients included in previous trials reveals a very heterogeneous population. As an example, most of the patients included in the CARES trial were obese [30], while the FREED trial enrolled mainly normal-weight subjects with a significantly lower proportion of hyperlipemia [31, 32]. Clearer identification of the patient phenotype in which SUA might have a greater impact on mortality could avoid further confusion in the future. Noteworthy, the risk of CVM may increase with SUA levels lower than those currently used in clinical practice to define the risk of gout [24]. This is of utmost importance, since there is an urgent need to develop and implement prevention and treatment strategies (e.g., lifestyle programs, diets, and pharmacotherapies) to reduce the $\mathrm{CV}$ burden. Prospective clinical trials are advisable to investigate whether the benefit of lowering SUA based on a cardiovascular threshold portends any prognostic advantage.

\section{Limitations}

The present findings relied on a large sample size and extended follow-up, which enabled the accumulation of sufficient events for robust and reliable analysis, including hard endpoints. However, the study design is retrospective. As such, the results should be cautiously interpreted, because the risk of selection bias is high, and there might be unmeasured variables that could potentially influence the relationship between SUA levels and outcomes. As this limitation is common to many other reports analysing the relationship between SUA and CVM, prospective studies are much needed, including randomised trials with uratelowering drugs. Dietary intake assessment was not implemented in our study, and thus, we had no information about the type of food consumed that may affect SUA levels. The patients enrolled had relatively low levels of SUA (only $1157 / 9589$ [12\%] had SUA $>7 \mathrm{mg} / \mathrm{dL}$ ); therefore, our findings might underestimate the mortality risk in populations with higher SUA levels. We did not collect data about nonfatal events during the clinical follow-up. The URRAH study was composed of subjects of white ethnicity, which included a percentage of patients selected from Hypertension clinics, thus resulting in a heterogeneous population. Consequently, further studies are needed to confirm that the thresholds of SUA emerging from our analyses are valid also in other populations.

\section{Conclusions}

Results from the present study confirm that sex-specific SUA cut-offs lower than those commonly considered to be at risk of gout are significantly associated with an increased risk of CVM in patients with MS, and independently from other conventional cardiovascular risk factors. If confirmed by future prospective studies, our data might contribute to improving further risk discrimination and reclassification of subjects with MS, which are already at high risk of CVM.

Supplementary Information The online version contains supplementary material available at https://doi.org/10.1007/s00392-021-01815-0.

Funding Open access funding provided by Università di Pisa within the CRUI-CARE Agreement. This work has been conducted with an unrestricted grant from the Fondazione of the Italian Society of Hypertension (Grant: MIOL). 
Availability of data and material Datasets generated during and/or analysed during the current study are not publicly available but are available from the corresponding author on reasonable request.

\section{Compliance with ethical standards}

Conflict of interest All the authors have nothing to declare.

Ethics approval The study was performed according to the Declaration of Helsinki for Human Research.

Consent to participate The URRAH study only used data routinely collected within the context of hypertension clinics for clinical reasons. No extra tests or interventions were undertaken on patients, and there was no impact on patient care or outcome. Prior to initiation of the study, approval to data collection was obtained from the Ethical Committee of the coordinating centre (Division of Internal Medicine, University of Bologna). The processing of the patients' personal data collected in the study complied with the European Directive on the Privacy of Data and all data collected, stored and processed were anonymised.

Consent for publication All the authors consent to publication.

Open Access This article is licensed under a Creative Commons Attribution 4.0 International License, which permits use, sharing, adaptation, distribution and reproduction in any medium or format, as long as you give appropriate credit to the original author(s) and the source, provide a link to the Creative Commons licence, and indicate if changes were made. The images or other third party material in this article are included in the article's Creative Commons licence, unless indicated otherwise in a credit line to the material. If material is not included in the article's Creative Commons licence and your intended use is not permitted by statutory regulation or exceeds the permitted use, you will need to obtain permission directly from the copyright holder. To view a copy of this licence, visit http://creativecommons.org/licenses/by/4.0/.

\section{References}

1. Mach F, Baigent C, Catapano AL et al (2020) 2019 ESC/EAS Guidelines for the management of dyslipidaemias: lipid modification to reduce cardiovascular risk. Eur Heart J 41:111-188

2. Cosentino F, Grant PJ, Aboyans V et al (2020) 2019 ESC Guidelines on diabetes, pre-diabetes, and cardiovascular diseases developed in collaboration with the EASD. Eur Heart J 41:255-323. https://doi.org/10.1093/eurheartj/ehz486

3. Williams B, Mancia G, Spiering W et al (2018) 2018 ESC/ESH Guidelines for the management of arterial hypertension: The Task Force for the management of arterial hypertension of the European Society of Cardiology and the European Society of Hypertension. Eur Heart J 39:3021-3104. https://doi.org/10.1097/HJH

4. Bombelli M, Quarti-Trevano F, Tadic M et al (2018) Uric acid and risk of new-onset metabolic syndrome, impaired fasting glucose and diabetes mellitus in a general Italian population: data from the Pressioni Arteriose Monitorate e Loro Associazioni study. J Hypertens 36:1492-1498. https://doi.org/10.1097/HJH.00000 00000001721

5. Mottillo S, Filion KB, Genest J et al (2010) The metabolic syndrome and cardiovascular risk: a systematic review and meta-analysis. J Am Coll Cardiol 56:1113-1132. https://doi.org/10.1016/j. jacc.2010.05.034
6. Cannon PJ, Stason WB, Demartini FE et al (1966) Hyperuricemia in primary and renal hypertension. N Engl J Med 275:457-464. https://doi.org/10.1056/NEJM196609012750902

7. Lehto S, Niskanen L, Rönnemaa T, Laakso M (1998) Serum uric acid is a strong predictor of stroke in patients with noninsulin-dependent diabetes mellitus. Stroke 29:635-639. https ://doi.org/10.1161/01.STR.29.3.635

8. Tuttle KR, Short RA, Johnson RJ (2001) Sex differences in uric acid and risk factors for coronary artery disease. Am J Cardiol 87:1411-1414. https://doi.org/10.1016/S0002-9149(01)01566 $-1$

9. Ford ES, Li C, Cook S, Choi HK (2007) Serum concentrations of uric acid and the metabolic syndrome among US children and adolescents. Circulation 115:2526-2532. https://doi.org/10.1161/ CIRCULATIONAHA.106.657627

10. Desideri G, Virdis A, Casiglia E et al (2018) Exploration into uric and cardiovascular disease: uric acid right for heart health (URRAH) project, a study protocol for a retrospective observational study. High Blood Press Cardiovasc Prev 25:197-202. https ://doi.org/10.1007/s40292-018-0250-7

11. Levey AS, Stevens LA, Schmid CH et al (2009) A new equation to estimate glomerular filtration rate. Ann Intern Med 150:604-612. https://doi.org/10.7326/0003-4819-150-9-200905050-00006

12. Cleeman JI (2001) Executive summary of the third report of the National Cholesterol Education Program (NCEP) expert panel on detection, evaluation, and treatment of high blood cholesterol in adults (adult treatment panel III). J Am Med Assoc 285:24862497. https://doi.org/10.1001/jama.285.19.2486

13. Sundström J, Byberg L, Gedeborg R et al (2011) Useful tests of usefulness of new risk factors: tools for assessing reclassification and discrimination. Scand J Public Health 39:439-441. https:// doi.org/10.1177/1403494810396556

14. Virdis A, Masi S, Casiglia E et al (2020) Identification of the uric acid thresholds predicting an increased total and cardiovascular mortality over 20 years. Hypertension. https://doi.org/10.1161/ HYPERTENSIONAHA.119.13643

15. Alberti KGMM, Eckel RH, Grundy SM et al (2009) Harmonising the metabolic syndrome: a joint interim statement of the international diabetes federation task force on epidemiology and prevention; National heart, lung, and blood institute; American heart association; World heart federation; International. Circulation 120:1640-1645

16. Galvan AQ, Natali A, Baldi $S$ et al (1995) Effect of insulin on uric acid excretion in humans. Am J Physiol Endocrinol Metab. https ://doi.org/10.1152/ajpendo.1995.268.1.e1

17. England LJ, Catalano PM, Levine RJ et al (2004) Glucose tolerance and risk of gestational diabetes mellitus in nulliparous women who smoke during pregnancy. Am J Epidemiol 160:12051213. https://doi.org/10.1093/aje

18. Masuo K, Kawaguchi H, Mikami H et al (2003) Serum uric acid and plasma norepinephrine concentrations predict subsequent weight gain and blood pressure elevation. Hypertension 42:474480. https://doi.org/10.1161/01.HYP.0000091371.53502.D3

19. Dehghan A, Van Hoek M, Sijbrands EJG et al (2008) High serum uric acid as a novel risk factor for type 2 diabetes. Diabetes Care 31:361-362. https://doi.org/10.2337/dc07-1276

20. Sánchez-Lozada LG, Tapia E, Bautista-García P et al (2008) Effects of febuxostat on metabolic and renal alterations in rats with fructose-induced metabolic syndrome. Am J Physiol Ren Physiol. https://doi.org/10.1152/ajprenal.00454.2007

21. Mercuro G, Vitale C, Cerquetani E et al (2004) Effect of hyperuricemia upon endothelial function in patients at increased cardiovascular risk. Am J Cardiol 94:932-935. https://doi.org/10.1016/j. amjcard.2004.06.032

22. Sautin YY, Nakagawa T, Zharikov S, Johnson RJ (2007) Adverse effects of the classic antioxidant uric acid in adipocytes: NADPH 
oxidase-mediated oxidative/nitrosative stress. Am J Physiol Cell Physiol. https://doi.org/10.1152/ajpcell.00600.2006

23. Packer M, Lam CSP, Lund LH et al (2020) Characterisation of the inflammatory-metabolic phenotype of heart failure with a preserved ejection fraction: a hypothesis to explain influence of sex on the evolution and potential treatment of the disease. Eur J Heart Fail 22:1551-1567

24. Selvaraj S, Claggett BL, Pfeffer MA et al (2020) Serum uric acid, influence of sacubitril-valsartan, and cardiovascular outcomes in heart failure with preserved ejection fraction: PARAGON-HF. Eur J Heart Fail 22:2093-2101. https://doi.org/10.1002/ejhf.1984

25. Krishnan E, Baker JF, Furst DE, Schumacher HR (2006) Gout and the risk of acute myocardial infarction. Arthritis Rheum 54:26882696. https://doi.org/10.1002/art.22014

26. Borghi C, Rosei EA, Bardin T et al (2015) Serum uric acid and the risk of cardiovascular and renal disease. J Hypertens 33:1729_ 1741. https://doi.org/10.1097/HJH.0000000000000701

27. Wang L, Zhang T, Liu Y et al (2020) Association of serum uric acid with metabolic syndrome and its components: a mendelian randomization analysis. Biomed Res Int. https://doi. org/10.1155/2020/6238693

28. Li X, Meng X, Timofeeva M et al (2017) Serum uric acid levels and multiple health outcomes: Umbrella review of evidence from observational studies, randomised controlled trials, and Mendelian randomisation studies. BMJ 357:j2376

29. Palmer TM, Nordestgaard BG, Benn M et al (2013) Association of plasma uric acid with ischaemic heart disease and blood pressure: mendelian randomisation analysis of two large cohorts. BMJ. https://doi.org/10.1136/bmj.f4262

30. White WB, Saag KG, Becker MA et al (2018) Cardiovascular safety of febuxostat or allopurinol in patients with gout. N Engl J Med 378:1200-1210. https://doi.org/10.1056/NEJMoa1710895

31. Kojima S, Matsui K, Hiramitsu S et al (2019) Febuxostat for cerebral and cardiorenovascular events prevention study. Eur Heart J 40:1778-1786A. https://doi.org/10.1093/eurheartj/ehz119

32. Kimura K, Hosoya T, Uchida S et al (2018) Febuxostat therapy for patients with stage $3 \mathrm{CKD}$ and asymptomatic hyperuricemia: a randomised trial. Am J Kidney Dis 72:798-810. https://doi. org/10.1053/j.ajkd.2018.06.028

\section{Author and Affiliations}

Nicola Riccardo Pugliese ${ }^{1}(1) \cdot$ Alessandro Mengozzi ${ }^{1} \cdot$ Agostino Virdis $^{1} \cdot$ Edoardo Casiglia $^{15} \cdot$ Valerie Tikhonoff $^{14}$. Arrigo F. G. Cicero ${ }^{2}$. Andrea Ungar ${ }^{4} \cdot$ Giulia Rivasi $^{4} \cdot$ Massimo Salvetti $^{3}$. Carlo M. Barbagallo ${ }^{16} \cdot$ Michele Bombelli $^{10}$. Raffaella Dell'Oro ${ }^{10}$. Berardino Bruno ${ }^{6}$. Luciano Lippa ${ }^{7}$. Lanfranco D'Elia ${ }^{11}$. Paolo Verdecchia ${ }^{17}$. Francesca Mallamaci ${ }^{18} \cdot$ Massimo Cirillo $^{13} \cdot$ Marcello Rattazzi $^{19} \cdot$ Pietro Cirillo $^{20} \cdot$ Loreto Gesualdo $^{20}$. Alberto Mazza ${ }^{21}$. Cristina Giannattasio ${ }^{22}$ - Alessandro Maloberti ${ }^{22} \cdot$ Massimo Volpe $^{23,24}$. Giuliano Tocci ${ }^{23,24}$. Georgios Georgiopoulos ${ }^{25}$. Guido laccarino ${ }^{12}$. Pietro Nazzaro ${ }^{26}$. Gianfranco Parati ${ }^{8,9}$. Paolo Palatini ${ }^{15}$. Ferruccio Galletti ${ }^{11}$. Claudio Ferri ${ }^{6}$. Giovambattista Desideri ${ }^{6}$. Francesca Viazzi ${ }^{5}$. Roberto Pontremoli ${ }^{5}$. Maria Lorenza Muiesan ${ }^{3}$. Guido Grassi ${ }^{10}$. Stefano Masi ${ }^{1}$. Claudio Borghi ${ }^{2}$. The Working Group on Uric Acid and Cardiovascular Risk of the Italian Society ofHypertension

8 Department of Cardiovascular, Neural and Metabolic Sciences, Istituto Auxologico Italiano, IRCCS S. Luca Hospital, Lucca, Italy

9 Department of Medicine and Surgery, University of Milan-Bicocca, Milan, Italy

10 Clinica Medica, Department of Medicine and Surgery, University of Milano-Bicocca, Monza, Italy

11 Department of Clinical Medicine and Surgery, University of Naples 'Federico II', Naples, Italy

12 Department of Advanced Biomedical Sciences, University of Naples 'Federico II', Naples, Italy

13 Department of Public Health, University of Naples 'Federico II', Naples, Italy

14 Department of Medicine and Studium Patavinum, University of Padua, Padua, Italy

15 Department of Medicine, University of Padua, Padua, Italy

16 Biomedical Department of Internal Medicine and Specialistics, University of Palermo, Palermo, Italy

17 Hospital S. Maria Della Misericordia, Perugia, Italy

18 Clinical Epidemiology of Renal Diseases and Hypertension, Reggio Cal Unit, CNR-IFC, Reggio Calabria, Italy
19 Department of Medicine, Medicina Interna $1^{\circ}, \mathrm{Ca}$ ' Foncello University Hospital, University of Padova, Treviso, Italy

20 Department of Emergency and Organ TransplantationNephrology, Dialysis and Transplantation Unit, Aldo Moro University of Bari, Bari, Italy

21 Department of Internal Medicine, Hypertension Unit, General Hospital, Rovigo, Italy

22 Cardiology IV, A. De Gasperis Department, Health Science Department, Niguarda Ca' Granda Hospital, Milano-Bicocca University, Milan, Italy

23 Hypertension Unit, Division of Cardiology, Department of Clinical and Molecular Medicine, Faculty of Medicine and Psychology, Sant'Andrea Hospital, University of Rome Sapienza, Rome, Italy

24 IRCCS Neuromed, Pozzilli, IS, Italy

25 First Department of Cardiology, Medical School, Hippokration Hospital, University of Athens, Athens, Greece

26 Department of Medical Basic Sciences, Neurosciences and Sense Organs, University of Bari Medical School, Bari, Italy 